\title{
Association between sleep quality and cardiovascular health: a door-to-door survey in rural Ecuador
}

\author{
Oscar H. Del Brutto $\cdot$ Robertino M. Mera • \\ Mauricio Zambrano • Victor J. Del Brutto • \\ Pablo R. Castillo
}

Received: 7 January 2014 / Accepted: 16 January 2014/Published online: 30 January 2014

(C) The Japanese Society for Hygiene 2014

\begin{abstract}
Objectives Cardiovascular correlates of sleep disorders have not been well investigated in underserved populations. The aim of this door-to-door survey was to evaluate the association between sleep quality and cardiovascular health status in persons aged $\geq 40$ years living in a village that is representative of rural Ecuador.

Methods All stroke-free Atahualpa residents aged $\geq 40$ years were screened with a validated Spanish version of the Pittsburgh Sleep Quality Index and the seven cardiovascular health $(\mathrm{CVH})$ metrics proposed by the American Heart Association to assess the CVH status.
\end{abstract}

O. H. Del Brutto and P. R. Castillo contributed uniformly to this work.

O. H. Del Brutto

School of Medicine, Universidad Espíritu Santo - Ecuador,

Guayaquil, Ecuador

O. H. Del Brutto $\cdot$ R. M. Mera - M. Zambrano ·

V. J. Del Brutto - P. R. Castillo

Department of Neurology, Hospital-Clínica Kennedy,

Guayaquil, Ecuador

O. H. Del Brutto $(\square)$

Air Center 3542, PO Box 522970, Miami, FL 33152-2970, USA

e-mail: oscardelbrutto@hotmail.com

\section{R. M. Mera}

Gastroenterology Department, Vanderbilt University,

Nashville, TN, USA

M. Zambrano - V. J. Del Brutto

Community Center, The Atahualpa Project, Atahualpa, Ecuador

P. R. Castillo

Sleep Disorders Center, Mayo Clinic College of Medicine,

Jacksonville, FL, USA
Results A total of 635 persons (mean age $59 \pm 13$ years; $58 \%$ women) were identified. A poor sleep quality was present in $27 \%$ of the subjects (95\% CI 24-31\%) and a poor cardiovascular health in $69 \%$ (95\% CI 63-71\%). In a multivariate logistic regression model, a poor sleep quality was associated with some $\mathrm{CVH}$ metrics in the poor range, including smoking status $(p=0.026)$, physical activity $(p=0.01)$ and blood glucose levels $(p=0.036)$. Conclusions A poor sleep quality may be linked to individual cardiovascular risk factors in this underserved Latin American population. This finding provides useful insights for the implementation of regional prevention priorities.

Keywords Cardiovascular health - Sleep quality - Sleep disorders · Population-based study $\cdot$ Ecuador · Atahualpa

\section{Introduction}

Sleep disorders not only interfere with the quality of life, but may trigger or worsen a number of conditions that are detrimental to health. Some studies have shown an association between sleep disorders and increased prevalence of cardiovascular risk factors and higher incidence rates of vascular outcomes and deaths [1]. Most of them, however, have focused on risks associated with sleep-disordered breathing and little attention has been paid to subjective sleep symptoms, such as the quality of sleep. A poor sleep quality has been recently associated with cognitive impairment in Japanese elders [2], and a national US survey showed no association between sleep quality and arterial hypertension [3]. Cardiovascular correlates of poor sleep quality have not been investigated in underserved populations living in the developing world. Lack of awareness on the importance of this association may be one 
of the factors responsible for the increasing burden of vascular events in rural areas of Latin America.

The Atahualpa Project is a population-based study primarily designed to evaluate cardiovascular risk factors and their correlates in rural coastal Ecuador [4]. Basal results have shown that Atahualpa residents have a better cardiovascular health $(\mathrm{CVH})$ status but similar stroke prevalence than their ethnic counterparts living in US [5]. As this paradox may be explained by the occurrence of non-traditional risk factors not well evaluated so far, we assess sleep quality and its cardiovascular correlates in this Ecuadorian Native/Mestizo population.

\section{Methods}

Methodology of this part of the Atahualpa Project (ClinicalTrials.gov identified: NCT01877616) has been detailed elsewhere [6]. The IRB of Hospital-Clínica Kennedy, Guayaquil, Ecuador (FWA 00006867), approved the protocol and the informed consent that had to be signed by all participants before entering the study. Trained field personnel performed a door-to-door survey to evaluate the quality of sleep and the CVH status of all stroke-free Atahualpa residents aged $\geq 40$ years. Sleep quality was assessed by the use of a validated Spanish version of the Pittsburgh Sleep Quality Index (PSQI) [7] and for CVH status evaluation, we used the seven CVH metrics proposed by the American Heart Association [8]. To recognize and exclude persons with stroke, all these persons were screened by rural doctors with the use of a validated field questionnaire, and then, certified neurologists evaluated suspected cases, as well as a random sample of negative individuals matched by age and sex for each positive case [9].

The PSQI discriminates between "good" and "poor" sleepers. Time-frame of such evaluation is in the month before the test. It consists of 19 items grouped into seven components, each weighted on a 0-3 scale, for a total score of 21 , with a score of $\geq 6$ indicating a poor sleep quality. Components include assessment of sleep duration, sleep disturbances, sleep latency, daytime dysfunction due to sleepiness, sleep efficiency, overall sleep quality, and medications needed to sleep [10].

The seven CVH metrics used to evaluate the CVH status included smoking status, body mass index (BMI), physical activity, diet, blood pressure, fasting glucose, and total cholesterol levels [8]. Each of these metrics was categorized as "ideal", "intermediate", and "poor", and the CVH status of the person was classified as poor if at least one metric was in the poor range.

Statistical analyses were carried out by using STATA version 13 (College Station, TX, USA). Descriptive
Table 1 Cardiovascular health $(\mathrm{CVH})$ status and metrics in strokefree Atahualpa residents aged $\geq 40$ years according to their quality of sleep

\begin{tabular}{|c|c|c|c|c|}
\hline & $\begin{array}{l}\text { Total } \\
\text { series } \\
(n=635)\end{array}$ & $\begin{array}{l}\text { Good } \\
\text { sleep } \\
\text { quality } \\
(n=462)\end{array}$ & $\begin{array}{l}\text { Poor } \\
\text { sleep } \\
\text { quality } \\
(n=173)\end{array}$ & $p$ value ${ }^{*}$ \\
\hline Age, mean \pm SD & $59 \pm 13$ & $57 \pm 11$ & $63 \pm 14$ & 0.001 \\
\hline Women $(\%)$ & 58 & 58 & 57 & 0.820 \\
\hline Poor CVH (\%) & 68.7 & 67.3 & 72.3 & 0.233 \\
\hline $\begin{array}{l}\text { Poor smoking status } \\
(\%)\end{array}$ & 1.9 & 1.3 & 3.5 & 0.086 \\
\hline $\begin{array}{l}\text { Poor body mass } \\
\text { index }(\%)\end{array}$ & 24.7 & 25.3 & 23.1 & 0.567 \\
\hline $\begin{array}{l}\text { Poor physical } \\
\text { activity (\%) }\end{array}$ & 4.7 & 2.6 & 10.4 & 0.001 \\
\hline Poor diet $(\%)$ & 3.6 & 3.9 & 2.9 & 0.547 \\
\hline $\begin{array}{l}\text { Poor blood pressure } \\
\text { (\%) }\end{array}$ & 35.1 & 33.8 & 38.7 & 0.244 \\
\hline $\begin{array}{l}\text { Poor blood glucose } \\
\text { levels }(\%)\end{array}$ & 28.7 & 26 & 35.8 & 0.015 \\
\hline $\begin{array}{l}\text { Poor total } \\
\text { cholesterol blood } \\
\text { levels }(\%)\end{array}$ & 11.2 & 11.9 & 9.2 & 0.346 \\
\hline
\end{tabular}

* Univariate logistic regression model, after adjusting for age and sex

statistics were presented as means with standard deviations for continuous variables and as percentages for categorical variables. A $p$ value of $<0.05$ was considered significant. Univariate and multivariate logistic regression analysis were used to study the relationship between PSQI positivity and $\mathrm{CVH}$ status as well as with each of the seven $\mathrm{CVH}$ metrics after adjusting for age and sex. All variables were dichotomized and the output of the model was the odds of a given relationship.

\section{Results}

The census identified a total of 688 Atahualpa residents aged $\geq 40$ years. Twenty-six persons declined to participate and 27 were further excluded because of a diagnosis of stroke. Therefore, sleep quality and $\mathrm{CVH}$ status were evaluated in 635 persons (mean age $59 \pm 13$ years; $58 \%$ women). Overall, 173 persons (27\%; $95 \%$ CI 24-31\%) had a poor sleep quality, defined as a score $\geq 6$ in the PSQI. Persons with poor sleep quality were older than those with a good sleep quality (mean \pm SD age: $63 \pm 14$ vs. $57 \pm 11$ years, $p<0.001)$; however, the percentage of women was the same across both groups (57 vs. $58 \%$ ). A poor $\mathrm{CVH}$ was noticed in 436 persons $(69 \%$; $95 \% \mathrm{CI}$ 63-71\%). Persons with a poor $\mathrm{CVH}$ were older than those with intermediate/ideal $\mathrm{CVH}$ (mean $\pm \mathrm{SD}$ age: $60.4 \pm 12.7$ vs. $55.9 \pm 11.4$ years, $p<0.001$ ), but there 
Table 2 Multivariate logistic regression model for the relationship between a poor sleep quality and each of the CVH metrics in 635 Atahualpa residents aged $\geq 40$ years (total $\mathrm{CVH}$ status was not included because of collinearity)

\begin{tabular}{llll}
\hline & $\begin{array}{c}\text { Odds } \\
\text { ratio }\end{array}$ & $95 \%$ CI & $p$ value \\
\hline Poor smoking status $(1.9 \%)$ & 3.90 & $1.18-12.90$ & 0.026 \\
Poor body mass index $(24.7 \%)$ & 0.92 & $0.59-1.42$ & 0.698 \\
Poor physical activity $(4.7 \%)$ & 2.89 & $1.29-6.46$ & 0.010 \\
Poor diet (3.6 \%) & 0.63 & $0.22-1.83$ & 0.399 \\
Poor blood pressure $(35.1 \%)$ & 0.90 & $0.60-1.34$ & 0.605 \\
Poor blood glucose levels $(28.7 \%)$ & 1.51 & $1.03-2.23$ & 0.036 \\
$\begin{array}{l}\text { Poor total cholesterol blood levels } \\
(11.2 \%)\end{array}$ & 0.77 & $0.42-1.41$ & 0.400 \\
\hline
\end{tabular}

was no difference in the percentage of women across both groups (56\% vs. $61 \%, p=0.286$ ).

In univariate logistic regression model, and after adjusting for age and sex, a poor sleep quality was not associated with a poor CVH status (OR 1.26; $95 \%$ CI $0.86-1.86 ; p=0.233)$. However, a poor sleep quality was significantly associated with some individual CVH metrics in the poor range, including physical activity (OR 4.35; $95 \%$ CI 2.05-9.25; $p<0.001)$ and blood glucose levels (OR 1.59; $95 \%$ CI $1.09-2.31 ; p=0.015$ ), and with a nonsignificant trend for smoking status (OR 2.73; $95 \%$ CI $0.87-8.58 ; p=0.086$ ) (Table 1). Multivariate analysis showed a significant positive correlation between poor sleep quality and poor smoking status, physical activity and blood glucose levels (Table 2).

\section{Discussion}

This study shows a significant increase in the prevalence of some individual $\mathrm{CVH}$ metrics in the poor range-namely smoking, physical activity, and blood glucose levels-in persons with a poor sleep quality, which persisted after adjusting for age and sex. The percentage of persons with a poor CVH status across good and poor sleepers was not different, however, and this could be related to the stringent criteria used by the American Heart Association for defining a person with poor CVH (only one metric in the poor range).

A previous study demonstrated that short sleep duration was associated with a high prevalence of the metabolic syndrome [11]. However, sleep duration based on a single self-reported "number of sleep hours" can be subject to sleep misperception and recall bias. This is only one of the several items of the PSQI, which is a better way for assessing the actual quality of sleep.
The relationship between sleep quality and $\mathrm{CVH}$ is complex and probably bidirectional. Unhealthy sleep patterns may alter cortisol release and affect the sympathetic nervous system which, in turn, may lead to increased cardiovascular risk factors [12]. On the other hand, bad habits, such as smoking and poor physical activity, may interfere with the quality of sleep [13]. A poor sleep quality among minorities may contribute to health disparities and may explain the increasing incidence of cerebrovascular diseases in rural areas of Latin America.

As we assessed sleep quality and CVH status in a crosssectional survey, we cannot be sure about causality. While this could be perceived as a potential weakness of our study, the homogeneous characteristics of Atahualpa's residents regarding race/ethnicity and socio-economic status, together with the strict criteria used for evaluation of sleep quality and $\mathrm{CVH}$ and the model used for assessing differences in $\mathrm{CVH}$ across good and poor sleepers, argues for the strength of our results. Further longitudinal studies in these underserved populations are warranted to settle the cause-and-effect relationships of this association. In addition, it is difficult to determine the precise basis for the reports of poor sleep quality without physiologic analyses. Future studies should include direct measures of sleep architecture and duration that only polysomnography and actigraphy may provide [6].

Acknowledgments This study was partially supported by an unrestricted grant from Universidad Espíritu Santo - Ecuador, Guayaquil Ecuador.

Conflict of interest The authors declare that they have no conflicts of interest.

\section{References}

1. Watson NF, Viola-Saltzman M. Sleep and comorbid neurologic disorders. Continuum (Minneap Minn). 2013;19:148-69.

2. Miyata S, Noda A, Iwamoto K, Kawano N, Okuda M, Ozaki N. Poor Sleep quality impairs cognitive performance in older adults. J Sleep Res. 2013;22:535-41.

3. Bansil P, Kuklina EV, Merritt RK, Yoo PW. Association between sleep disorders, sleep duration, quality of sleep, and hypertension: results from the National Health and Nutrition Examination Survey, 2005 to 2008. J Clin Hypertens. 2011;13:739-43.

4. Del Brutto OH, Peñaherrera E, Ochoa E, Santamaría M, Zambrano M, Del Brutto VJ. Door-to-door survey of cardiovascular Health, stroke, and ischemic Heart disease in rural coastal Ecuador: the Atahualpa Project: methodology and operational definitions. Int J Stroke. 2013; doi:10.1111/ijs.12030.

5. Del Brutto OH, Dong C, Rundek T, Elkind MSV, Del Brutto VJ, Sacco RL. Cardiovascular health status among Caribbean Hispanics living in Northern Manhattan and Ecuadorian Native/ Mestizos in rural coastal Ecuador: a comparative study. J Community Health. 2013;38:634-41.

6. del Brutto $\mathrm{OH}$, Mera RM, Farfán R, Castillo PR. Cerebrovascular correlates of sleep disorders: rational and protocol of a 
door-to-door survey in rural coastal Ecuador. J Stroke Cerebrovasc Dis. 2013;. doi:10.1016/j.strokecerebrovasdis.2013.08.020.

7. Jiménez-Genchi A, Monteverde-Maldonado E, Nenclares-Portocarrero A, Esquivel-Adame G, de la Vega-Pacheco A. Confiabilidad y análisis factorial de la versión en español del índice de calidad de sueño de Pittsburgh en pacientes psiquiátricos. Gac Med Mex. 2008;144:491-6.

8. Lloyd-Jones D, Hong Y, Labarthe D, Mozaffarian D, Appel LJ, Van Horn L, et al. American Heart Association strategic planning task force and statistics committee. Defining and setting national goals for cardiovascular health promotion. The American Heart Association's strategic impact goal through 2020 and beyond. Circulation. 2010;121:586-613.

9. Del Brutto OH, Santamaría M, Zambrano M, Peñaherrera E, Pow-Chon-Long F, Del Brutto VJ, et al. Stroke in rural coastal
Ecuador: a community-based survey. Int J Stroke. 2013;. doi:10. 1111/ijs.12102.

10. Buysee DJ, Reynolds CF 3rd, Monk TH, Berman SR, Kupfer DJ. The Pittsburgh sleep quality index: a new instrument for psychiatric practice and research. Psychiatry Res. 1989;28:193-213.

11. Jennings JR, Muldoon MF, Hall M, Buysse DJ, Manuck SB. Selfreported sleep quality is associated with the metabolic syndrome. Sleep. 2007;30:219-23.

12. Gangwisch JE. Epidemiological evidence for the links between sleep, circadian rhythms, and metabolism. Obes Rev. 2009;10(Suppl 2):37-45.

13. Choi SH, Redman RW, Terrell JE, Pohl JM, Duffy SA. Factors associated with health-related quality of life among operating engineers. J Occup Environ Med. 2012;54:1400-5. 